\title{
Stochastic nonlinear multiscale computational scheme for short fiber reinforced composites to study the influence of microstructural variability on damage propagation
}

\author{
Tien-Dat Hoang ${ }^{1,2}\left(\mathbb{D} \cdot\right.$ Yutaro Abe $^{1} \cdot$ Shinya Nakamura $^{3} \cdot$ Akio Miyoshi $^{3} \cdot$ Naoki Takano $^{4}$
}

Received: 14 October 2019 / Accepted: 3 January 2020 / Published online: 10 January 2020

(c) Springer Nature Switzerland AG 2020

\begin{abstract}
In this paper, a stochastic nonlinear multiscale computational scheme is proposed to study the influence of the variability in both physical parameters of constituent materials and geometrical parameters such as fiber orientation, fiber arrangement of short fiber reinforced composites on the damage propagation. The aim is to predict many probable damage patterns in a huge number of scenarios under complex strain conditions and to discuss the influential level of variability in the physical and geometrical parameters for short fiber reinforced composites. The parameterization and numerical modelling for short fiber reinforced plastics made by injection molding are conducted. The random physical parameters are considered theoretically by the first-order perturbation based stochastic homogenization method, while sampling is used for the random geometrical parameters. When the scenario becomes huge in the stochastic nonlinear analysis, a computational scheme using sub-sampling is proposed. Moreover, the stochastic prediction of the homogenized properties of the composite material and their probable degradation are discussed. The stochastic computation of microscopic strains is analyzed though the damage propagation simulation. When the geometrical variability is considered, a definition of an average strain distribution among many samples is also presented. The efficiency of the accelerated element-by-element scaled conjugate gradient iterative solver is shown by solving many three-dimension random short fiber reinforced models with hundreds of thousands of degrees of freedom. The stochastic computational scheme provides a suitable reference to predict failure under specific reliability requirements of short fiber reinforced composites or other composite materials.
\end{abstract}

Keywords Stochastic finite element method - Sampling and sub-sampling · Variability · Microscopic damage evolution . Fiber reinforced composites

\section{Introduction}

All materials, especially composite materials, are characterized by various degrees of inherent variability or randomness. Variability exists on all scales from the arrangement of a material microstructure to the structure at the macroscale. Particularly, there may exist variability in the constituent material properties, and geometries of the composite materials at various scales. The variability in these parameters induces variability of the mechanical behavior and damage evolution in the materials which may cause severe random reflections of composite structures. Besides, the variability of the materials may result in huge unpredicted scenarios of damage evolution.

$\triangle$ Tien-Dat Hoang, hoangdat_tnut@keio.jp | ${ }^{1}$ Graduate School of Science and Technology, Keio University,

Kohoku-ku, Yokohama 223-8522, Japan. ${ }^{2}$ Faculty of International Training, Thai Nguyen University of Technology, Thai Nguyen 23000 ,

Vietnam. Insight, Inc, 5-29-12-407 Hongo, Bunkyo, Tokyo 113-0033, Japan. ${ }^{4}$ Department of Mechanical Engineering, Keio University, Kohoku-ku, Yokohama 223-8522, Japan. 
Damages in a composite structure may remain hidden below the surface, undetectable by visual inspection until the entire structure has failed. Recently, the stochastic finite element method (SFEM) is an efficient technique to consider variability or randomness in composite materials and has become a useful tool in several engineering fields. It is always required to develop a robust computation framework to take into account the variability or randomness in a design process of composite structures.

Some popular SFEMs, such as the spectral method [1] including the Karhunen-Loeve (KL) expansion [2], the polynomial chaos (PC) expansion [3], and the stochastic perturbation method [4-7], are used to represent the input and output random fields. Computational cost in SFEM is smaller than that in the Monte Carlo simulation (MCS), because the accuracy of MCS depends on the large enough number of samples, especially when there are many random parameters. The spectral method was used to represent the result in an explicit form, using a finite number of random variables. The $\mathrm{KL}$ and $\mathrm{PC}$ expansions consist of discretizing random parameters in a Fourier-like series and solve the problem along with a basis of orthogonal random variables. The stochastic perturbation technique is based on the $n$-th order Taylor expansion. Using the stochastic perturbation technique, Talha and Singh [6] analysed the thermo-mechanical stability with randomness in the material properties of functionally graded material plates. Zhou et al. [7] presented a perturbationbased stochastic homogenization framework to predict the effective elastic properties considering randomness in the macroscopic properties of the constituents. One of the authors introduced a first-order perturbation based stochastic homogenization (FPSH) method for random modelling in the estimation of mechanical properties of human vertebral trabecular bone [5]. Afterward, this method was applied to analyze three-phase composite material considering randomness in both physical and geometrical parameters at the microscale [8]. The FPSH method has been developed for considering many random physical parameters in constituent materials to clarify the effect of randomness in each engineering constant of constituent mechanical properties [9]. Additionally, the accuracy of the FPSH was verified and compared with the MCS. The asymptotic expansion homogenization method would be used to simulate the damage propagation of a brittle representative volume element (RVE) model with a low level of damage or failure probability at the micro scale $[10,11]$. The asymptotic homogenization approach was also clearly presented by Ramírez-Torres et al. [12]. Since to shorten the entire computational process in nonlinear analysis, and since the results of the higher-order expansion are not always give higher accuracy than that of the first-order expansion, the first-order perturbation technique was used our previous work [13] and in this work.

For the improvements in the design processing technologies on cost-efficient design, the building block approach (BBA) is applied to test scale-up from coupons, elements, and subcomponents to establish final composite structures $[14,15]$. At the lower level of the BBA, the composite microstructures of coupons are tested. The engineering constants, strengths, and strain-to-failure of a coupon are estimated by tension, compression, and shear tests [15]. However, the manufacturing processes of composite materials involves many randomness or variability in microstructures affecting the quality of the component. This BBA needs to involve many experiments to achieve a safe design. It results in excessive cost and time-consuming processes. Therefore, the replacement of a huge number of experimental tests of composite materials by the stochastic numerical simulation considering the variability or randomness is a big matter of concern recently.

The types of variabilities in SFEM can be categorized into the variabilities of physical [8], geometrical parameters $[16,17]$ and other sources such as initial/boundary conditions and loading conditions [18]. Particularly, the parameterization of geometrical variability is very important but challenging. One of the authors investigated the influence of the variability in geometrical parameters of fluctuated meso/micro-structure of plain-woven fabric GFRP laminate on its properties and introduced the parameterized geometrical quantities of randomness [17]. Recently, fiber reinforced composite materials have many applications in a wide range of engineering fields and industries such as aerospace, automobile, and light industrial products, because of their low density, high strength and design flexibility [19]. Especially, short fiber reinforced composites are being used extensively since they can be used to make large and complex body parts [20]. During the injection molding process, fibers breakage also usually occurs, and a random orientation distribution, a fiber length distribution, random fiber arrangement would result in the final product. Mortazavin and Faremi [19] investigated the anisotropic effects on tensile strength of short fiber reinforced plastics and found the variation of these materials caused by fiber orientation. loannis et al. [21] showed the larger effect of microstructure parameters such as aspect ratio, fiber orientation on the macroscopic behavior of short fiber reinforced thermoplastic composites. The mechanical properties of short fiber reinforced composites are critically dependent on these morphological structures, especially fiber orientation distribution which can result in the unreliable determination of the deterministic computation [22]. The fiber orientation in short fiber reinforced composites made by injection molding has been optimized by Chen et al. [23]. 
Additionally, fiber arrangement in microstructures of short fiber reinforced composites should also be considered. To maximize the effectiveness of short glass fiber structures in mechanical properties, damage behaviors, fiber orientation, and fiber arrangement were deeply considered in this research.

So far, a few researches were presented to investigate microscopic damage of fiber reinforced composites. For instance, Mishnaevsky et al. [24] proposed a numerical algorithm to investigate damage evolution and analyzed the interplay of damage mechanisms in unidirectional fiber reinforced composites. Sasayama et al. [25] introduced the tensile failure of injection molded short glass fiber reinforced polyamide 6,6 by using a multiscale mechanistic model. Notta-Cuvier et al. [26] presented a damage model for short fiber reinforced composites with random fiber orientation subjected to uniaxial tension. An analysis of fracture progress in unidirectional composites under tension using the extended finite element method was performed by Wang et al. [27]. Jha et al. [28] introduced a computational modelling framework for investigating the damage effects into fiber reinforced matrix composite materials. When considering variability or randomness in damage analysis, a nonlinear SFEM is recommended. Darith-Anthony Hun et al. [29] proposed a stochastic multiscale approach to model crack propagation in random heterogeneous microstructure and described the characterization of subscale-induced randomness on the macroscopic response of the domain. Karel Matouš et al. [30] showed the big role of virtual material testing and three-dimensional multiscale simulation for the nonlinear material behavior and the importance of multiscale computation to consider aleatoric uncertainty from the microscopic scale to the macroscopic scale.

In the following work, the influence of microstructural variability on the damage propagation of short fiber reinforced composites is investigated by a stochastic nonlinear multiscale computational scheme. In Sect. 2, microstructure modeling of a short fiber reinforced plastic made by injection molding is introduced. In addition to the FPSH method for considering random physical parameters is presented. Following that, the scheme of sampling using for random geometrical parameters, and scheme of sub-sampling using for a huge scenario in the stochastic nonlinear analysis are proposed. In Sect. 3, one numerical exampling to show the application of the proposed scheme for the short fiber reinforced plastic is carried out to predict probable damage patterns under a complex strain condition. The discussion of the influence of physical and geometrical parameters on this material is also shown in this section. The efficiency of the accelerated elementby-element scaled conjugate gradient (EBE-SCG) solver [13] is performed in this example. Conclusion remarks are provided in Sect. 4. This present work represents the first step towards developing a robust simulation framework for the prediction of practical damage evolution of composite materials.

\section{Stochastic nonlinear multiscale computational scheme}

\subsection{Microstructure modeling of short fiber reinforced composites}

In this section, a modelling scheme of short fiber reinforced composite materials is presented. When modelling, all geometrical parameters such as fiber orientation, fiber lengths, fiber arrangement, or fiber ratio should be considered. However, in this work, only fiber orientation and fiber arrangement, which may have significant effects on the properties and damaged behavior of the material, are investigated considering a certain fiber length distribution. Figure 1 shows the microstructure modeling for a skin-core-skin layered specimen of injection molded short fiber reinforced composite material with a two-scale coordinate setup with the definition of a scale ratio $\lambda$ of a macroscopic scale $x$ and a microscopic scale $y$ by Eq. (1). Microstructures of injection molded short fiber reinforced plastics can be divided into 3 regions including skin-coreskin regions [31]. Due to large shearing near the mold wall, the fibers in the skin regions are mainly aligned in the flow direction and are the dominant layers. In the core region, because of low shearing and large extensional flow, the fibers are more aligned transversely to the flow direction. The fibers in the first layer of the skin regions are immediately adjacent to the cavity wall due to the fountain flow, which moves and rotates the fibers toward the wall. The geometry of mold, the type of resin, and other injection

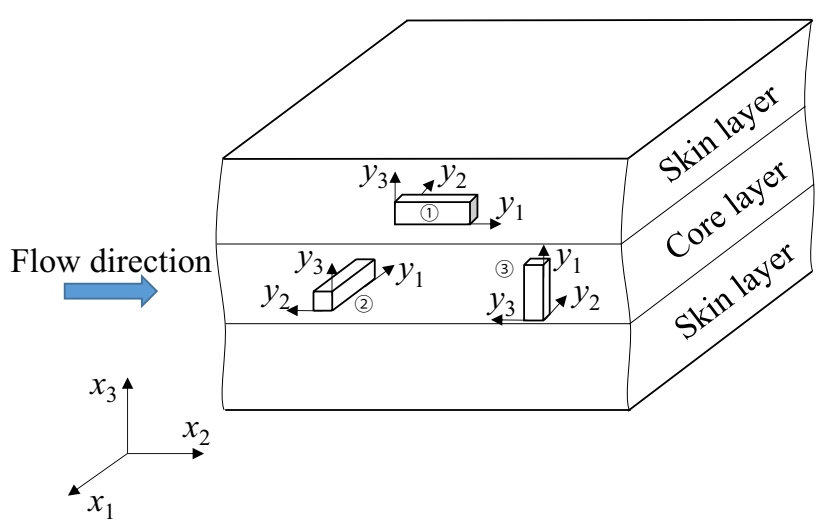

Fig. 1 Microstructure modeling for skin-core-skin layered specimen of injection molded short fiber reinforced composite material 
parameters lead to the variability of fiber orientation in the skin regions. As the basic building block approach of fiber reinforced composites, the investigation or prediction of microscopic damage propagation of injection molded products at the coupon level is very important to avoid over-designed structure and high-risk structural designs. The variability of the composite material can be captured in the microstructures of coupons by using statistical representative element (SVE) whose minimum size is decided based on statistics of nearest fiber distance, fiber length, fiber orientation and fiber volume fraction.

$\lambda=\frac{\boldsymbol{x}}{\boldsymbol{y}}$

In the mechanic approach, fiber orientation is modelled by using fiber orientation tensor [32]. On the other hand, in the manufacturing approach, fiber orientation is modelled by using two angles $\varphi$ and $\theta$ assigned in ranges of $\left[-\varphi_{\text {min' }} \varphi_{\text {max }}\right]$ and $\left[-\theta_{\text {min' }} \theta_{\text {max }}\right]$ as shown in Fig. 2 . Note that when $\varphi=0^{\circ}$ and $\theta=90^{\circ}$, all the fibers are oriented in the flow direction, whereas $\varphi=90^{\circ}$ and $\theta=90^{\circ}$ means the orientation is perpendicular to the flow direction. In this

Fig. 2 Definition of fiber orientation
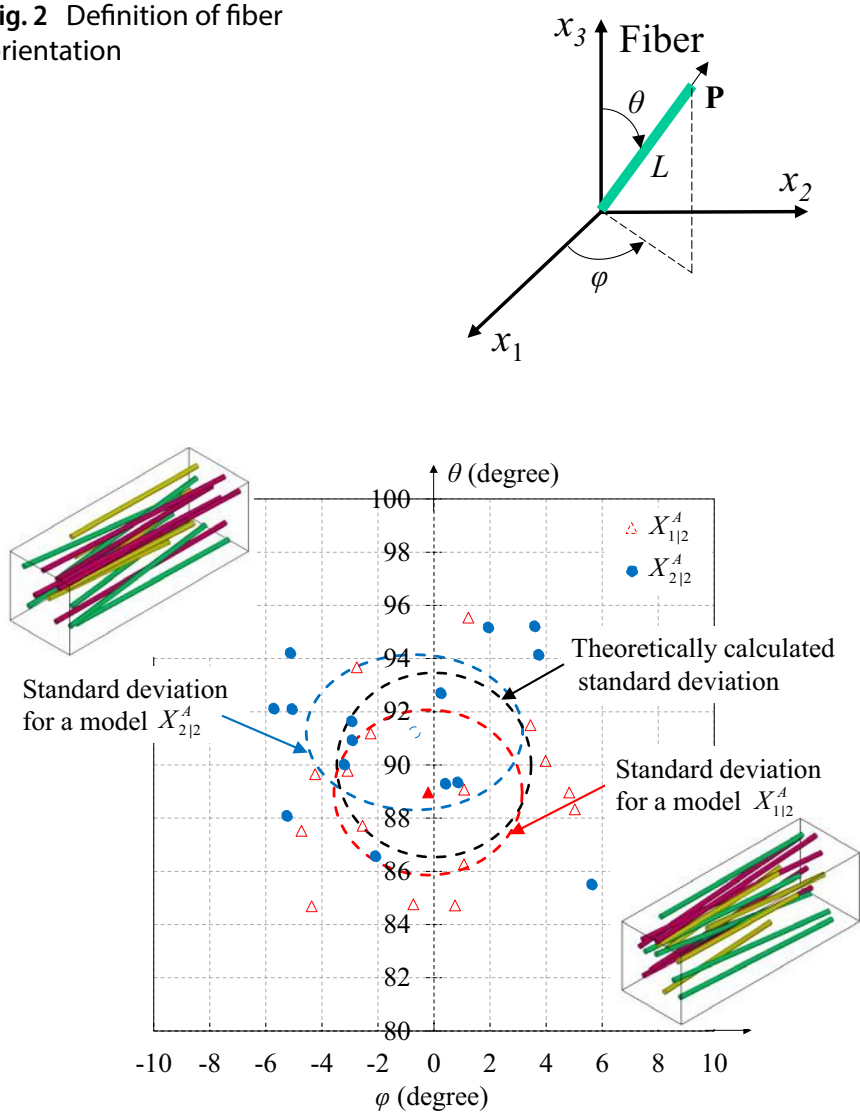

(a) $\varphi=[-6,6]$ and $\theta=[84,96]$ stochastic simulation, the fiber orientation sample $j$ is denoted by $X_{j}^{O}$ with random distribution $f\left(X_{j}^{O}\right)$. The fiber arrangement sample $i$ is denoted by $X_{i \mid j}^{A}$ with random distribution $f\left(X_{i \mid j}^{A}\right)$. Where $f$ denotes the probability density function. To automatically model random short fiber reinforced composites, we customized a commercial software Meshman Particle Packing (Insight, Inc., Tokyo, Japan) [33]. Meshman Particle Packing is a model generator for the discrete element method (DM). The software can pack not only isolated particles but also cluster particles in a specified region with arbitrary shapes. Therefore, we developed this software to generate random short fibers. In other words, this software includes an engine for packing short fibers using random numbers. We can set arbitrary lengths for short fibers, fiber orientations, and fiber arrangements as well. To this end, from input candidates of different fiber orientations, different fiber lengths, this software can easily generate many models with a different total number of fibers packed in the same region, and different fiber orientation distributions by random numbers. Additionally, it is one step to provide a tool to find the optimal three-dimension SVE size of not only short fiber reinforced plastics but also other complex composites.

During the injection molding process, the resin flow and complicated structure cause fibers to be non-uniformly dispersed in random orientation and random arrangement. For illustration of the modelling, Fig. 3 shows some microstructure models with different fiber arrangements for the given fiber orientation variabilities of 16 short fibers generated by the Meshman Particle Packing. All information on these models will be provided more in Sect. 3 .

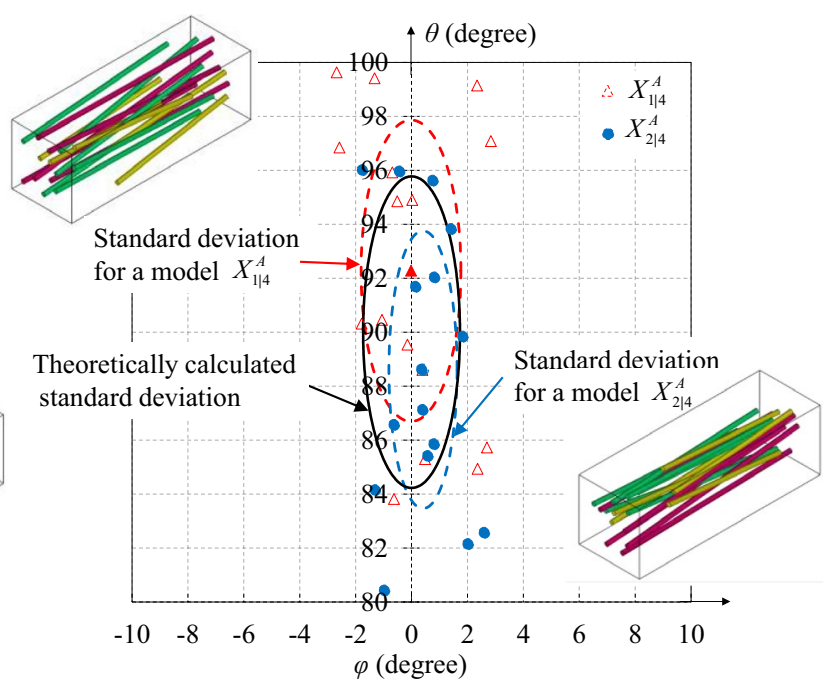

(b) $\varphi=[-3,3]$ and $\theta=[80,100]$

Fig. 3 Microstructure models with different fiber arrangements for given fiber angel variabilities by modified Meshman ParticlePacking 
When the physical and geometrical randomness at the microscale is considered, the prior probabilistic homogenization method is applied to many microstructure models according to fiber orientation sample $j$ and fiber arrangement sample $i$ in case of $j$. In the probabilistic procedure, the expectation is not only to find the output results from a single value of an arbitrary quantity of interest such as homogenized properties $\boldsymbol{D}^{H}$, microscopic strain $\varepsilon$ but also the random distribution in the quantities of interest $f\left(\boldsymbol{D}^{H}\right)$ and $f(\varepsilon)$. The probability density function of homogenized property and average strain for many SVEs considering randomness in both physical and geometrical parameters can be obtained by the Gaussian mixture distribution method as in Eqs. (2-3). A Gaussian mixture distribution is used, because it can capture nonlinear formulation and quantify the randomness on the results. It is also a probabilistic model that assumes all the results of many models are represented by a mixture of Gaussian distributions with random parameters [34]. In this work, $j_{\max }$ is the maximum number of fiber orientation samples and $i_{\text {max }}$ is the maximum number of fiber arrangement samples concerning $j$.

$f\left(\boldsymbol{D}^{H}\right)=\sum_{j}^{j_{\max }} f\left(X_{j}^{O}\right)\left\{\sum_{i}^{i_{\max }} f\left(X_{i \mid j}^{A}\right) f\left(\boldsymbol{D}_{X_{i \mid j}^{H}}\right)\right\}$

$\operatorname{Ave}(\varepsilon)=\sum_{j}^{j_{\max }} f\left(X_{j}^{O}\right)\left\{\sum_{i}^{i_{\max }} f\left(X_{i \mid j}^{A}\right) f\left(\varepsilon_{X_{i \mid j}}\right)\right\}$

where $\sum_{j}^{j_{\max }} f\left(X_{j}^{O}\right) \sum_{i}^{i_{\max }} f\left(X_{i \mid j}^{A}\right)=1 . f\left(X_{j}^{O}\right)$ and $f\left(X_{i \mid j}^{A}\right)$ are considered as the Gaussian weighted densities. They could be given first and then updated by experimental results which can be seen in our previously published paper [5]. In this work, they are obtained by taking flat probability. $f\left(\boldsymbol{D}_{X_{i \mid j}}^{H}\right)$ and $f\left(\varepsilon_{X_{i \mid j}}\right)$ will be derived and presented in the following part.

\subsection{First-order perturbation based stochastic homogenization (FPSH) method considering random physical parameters}

In the general formulation for a certain SVE, we consider a three-dimensional elastic body $\Omega$ as the assembly of periodic microstructures SVEs as shown in Fig. 4. The elastic constants vary within an SVE of the composite. The solution of displacements also depends both on $x$ and scale ratio $\lambda$. Dependence on $y$ can be considered periodic for a fixed $x$ at the macroscopic level. For different points $x$, the structure of the composite may vary, but on a point $x$, a periodic pattern can be found. The SVE model $Y$ is the union of each constituent material domain $Y_{r}$, where $r=1$,

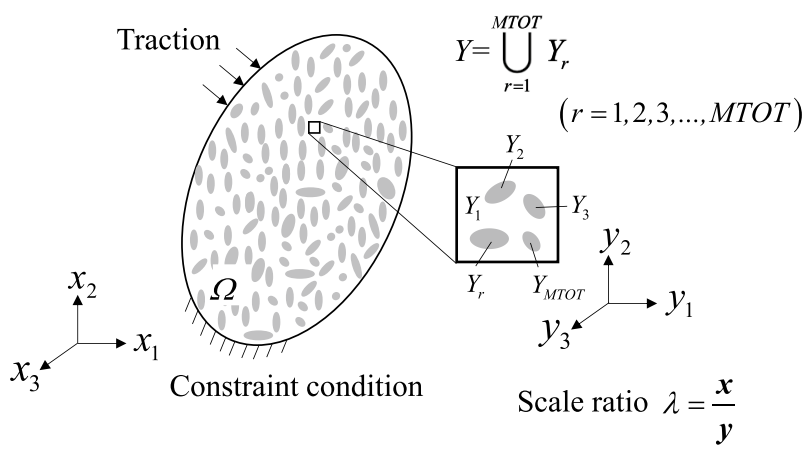

Fig. 4 Two-scale problem of heterogeneous media

$2,3, \ldots$, MTOT. The volume of $Y$ is defined by $|Y| . \Omega$ is a random quantity. The physical quantity is dependent on the microstructure. The displacement depends on both $x$ and $y$ of the domain $\Omega \times Y$. To analyse microscopic behaviours of composite materials at the coupon level, a two-scale homogenization method was studied [13]. The homogenized composite material model is utilized for the global mesh, while the microscopic heterogeneity, microscopic strain, and damage propagation are considered in the SVE model with random physical parameters of constituent materials and variabilities of geometrical parameters.

The macroscopic displacement is expanded asymptotically using the perturbation parameter $\lambda$ [13]. The perturbed displacement expresses the heterogeneous microstructure deformation. A characteristic displacement $\chi_{s}^{k l}$ of a periodic function with respect to the microscale $y$ was assumed in the same way as conventional homogenization theory [35]. It shows that the macroscopic strain can bridge the macroscale and microscale as well as the microscopic displacement. The Voigt notation $k l=11,22$, $33,23,31,12$ is used to show six modes of characteristic displacements and index $s=1-3$ shows the degree of freedom of each node. Each mode of the characteristic displacements $\chi^{\mathrm{kl}}$ shows the displacement under corresponding applied macroscopic strain due to the microscopic heterogeneity [13]. $\chi^{k l}$ also depends on the location of a point $x$. Additionally, characteristic displacements will be changed in nonlinear damage propagation solution due to the increase of given macroscopic strain, or loading conditions.

In the derivation of two-scale homogenization theory, an averaging principle for a periodic function $g$ used for the microscopic SVE model is given by Eq. (4) [35].

$\lim _{\lambda \rightarrow 0} \int_{\Omega} g\left(\frac{\boldsymbol{x}}{\lambda}\right) d \Omega=\int_{\Omega} \frac{1}{|Y|} \int_{Y} g(\boldsymbol{y}) d Y d \Omega$

The class of the model is characterized by the assumption that the macroscopic strain $\boldsymbol{E}$ and stress $\boldsymbol{\Sigma}$ at a point 
$x$ are the volume averages of their respective microscopic identical fields over a pre-described SVE as shown in Eq. (5). Where $\boldsymbol{\varepsilon}$ and $\boldsymbol{\sigma}$ are microscopic strain and stress, respectively.

$\boldsymbol{E} \equiv \frac{1}{|Y|} \int_{Y} \varepsilon d Y, \quad \Sigma \equiv \frac{1}{|Y|} \int_{Y} \sigma d Y$

Suppose that, in a heterogeneous material, the properties of constituents have randomness or variability. To express the randomness in the physical parameters of constituents, $\boldsymbol{\alpha}=\left(\alpha_{1}, \alpha_{2}, \ldots\right)$ is used. In this formulation, $f(\alpha)$ complies with a normal distribution corresponding to $\operatorname{Exp}(f(\alpha))=0$ and $\operatorname{Var}(f(\alpha))=\alpha^{2}$ [8]. Usually, $\alpha$ depends on $\lambda$ and therefore $\alpha$ is defined at the microscopic scale. Here- $f\left(\boldsymbol{u}^{\text {micro }}\right)=\boldsymbol{E} \boldsymbol{y}-f\left(\chi^{k l}\right) \boldsymbol{E}_{k l}$

Based on the above setup in Eq. (6), the characteristic displacement also can be expanded by the first-order perturbation with respect to $\alpha$ with the formula in Eq. (8) [13].

$f\left(\chi^{k l}\right) \approx\left(\chi^{k l}\right)^{0}+\sum_{r}^{\text {MTOT }} \sum_{m=1}^{6} \sum_{n=1}^{6}\left(\chi_{r, m n}^{k l}\right)^{1} f\left(\alpha_{r, m n}\right)$

For solving by finite element method, the zeroth, and first-order terms in a discretized form of $\chi^{k l}$ can be obtained by solving the Eqs. (9-10), respectively.

$\left(\int_{Y} \boldsymbol{B}^{T} \boldsymbol{D}^{0} \boldsymbol{B} d Y\right)\left(\chi^{k l}\right)^{0}=\int_{Y} \boldsymbol{B}^{T}\left(\boldsymbol{D}^{0} \boldsymbol{Q}^{k l}\right) d Y$

$\left(\int_{Y} \boldsymbol{B}^{T} \boldsymbol{D}^{0} \boldsymbol{B} d Y\right)\left(\chi_{r, m n}^{k l}\right)^{1}=\int_{Y} \boldsymbol{B}^{T}\left(\boldsymbol{P}_{m n} D_{r, m n}^{1}\right) \boldsymbol{Q}^{k l} d Y-\int_{Y} \boldsymbol{B}^{T}\left(\boldsymbol{P}_{m n} D_{r, m n}^{1}\right) \boldsymbol{B}\left(\chi^{k l}\right)^{0} d Y$

where $B$ is the strain-displacement matrix and an operator

after, vector and matrix form are used. It is assumed that microscopic mechanical properties of constituent materials vary corresponding to random physical parameters $\alpha$ according to the normal distribution as in Eq. (6).

$f\left(\boldsymbol{D}_{r}\right) \approx \boldsymbol{D}_{r}^{0}+\sum_{m=1}^{6} \sum_{n=1}^{6}\left(\boldsymbol{P}_{m n} D_{r, m n}^{1}\right) f\left(\alpha_{r, m n}\right)$

where $\boldsymbol{D}_{r}^{0}$ and second term expresses the expected values and variance of mechanical properties, and the operator $\boldsymbol{P}_{m n_{s t}}=\left\{\begin{array}{l}1 \text { for }(s, t)=(m, n),(n, m) \\ 0 \text { for others }\end{array}\right.$ component $m n$ of constituent material $r$ corresponding to its random physical parameter $\alpha_{r, m n}$ to the stress-strain matrix $\boldsymbol{D}_{r} . D_{r, m n}^{1}$ is the component $m n$ of the first order terms of $\boldsymbol{D}_{r}$. to extract a column from a matrix is defined as $\mathbf{Q}^{k l}=$

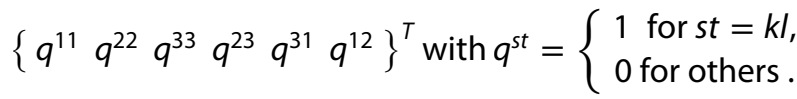

Subsequently, the macroscopic stress-strain matrix $\boldsymbol{D}^{H}$ can be computed by Eq. (11). The zeroth and first-order terms of $\boldsymbol{D}^{H}$ are obtained in Eqs. (12-13) by using all modes of the zeroth and first-order terms of $\chi^{k l}$.

$$
\begin{aligned}
& f\left(\boldsymbol{D}^{H}\right) \approx\left(\boldsymbol{D}^{H}\right)^{0}+\sum_{r}^{\text {MTOT }} \sum_{m=1}^{6} \sum_{n=1}^{6}\left(\boldsymbol{D}_{r, m n}^{H}\right)^{1} f\left(\alpha_{r, m n}\right) \\
& \left(\boldsymbol{D}^{H}\right)^{0}=\frac{1}{|Y|} \int_{Y}\left(\boldsymbol{D}^{0}-\boldsymbol{D}^{0} \boldsymbol{B}\left(\chi^{k l}\right)^{0}\right) d Y
\end{aligned}
$$

$\left(\boldsymbol{D}_{r, m n}^{H}\right)^{1}=\frac{1}{|Y|} \int_{Y}\left(\boldsymbol{P}_{m n} D_{r, m n}^{1}-\boldsymbol{P}_{m n} D_{r, m n}^{1} \boldsymbol{B}\left(\chi^{k l}\right)^{0}-\boldsymbol{D}^{0} \boldsymbol{B}\left(\chi_{r, m n}^{k l}\right)^{1}\right) d Y$

For a certain SVE, the microscopic displacement, which is different from the perturbed displacement, can be written by Eq. (7) [36]. Where the first term is associated with a homogeneous strain field corresponding to the macroscopic strain $\boldsymbol{E}$, and the second term is the displacement fluctuation field. Similarly, the SVE strain field can be decomposed into a homogeneous term and a fluctuation term. Because the volume average of this strain field is equal to the macroscopic strain, the volume average of the displacement fluctuation term or the strain fluctuation term needs to equal 0 [9].
When the given macroscopic strain $\boldsymbol{E}$ is applied to the SVE model, the microscopic strain $\boldsymbol{\varepsilon}$ of each element is determined in a stochastic way by Eq. (14).

$f(\boldsymbol{\varepsilon}) \approx\left(\boldsymbol{E}-\boldsymbol{B}\left(\chi^{k l}\right)^{0} \boldsymbol{E}\right)-\sum_{r}^{\text {MTOT }} \sum_{m=1}^{6} \sum_{n=1}^{6} \boldsymbol{B}\left(\chi_{r, m n}^{k l}\right)^{1} \boldsymbol{E} f\left(\alpha_{r, m n}\right)$ 
Fig. 5 Algorithm of stochastic damage propagation analysis

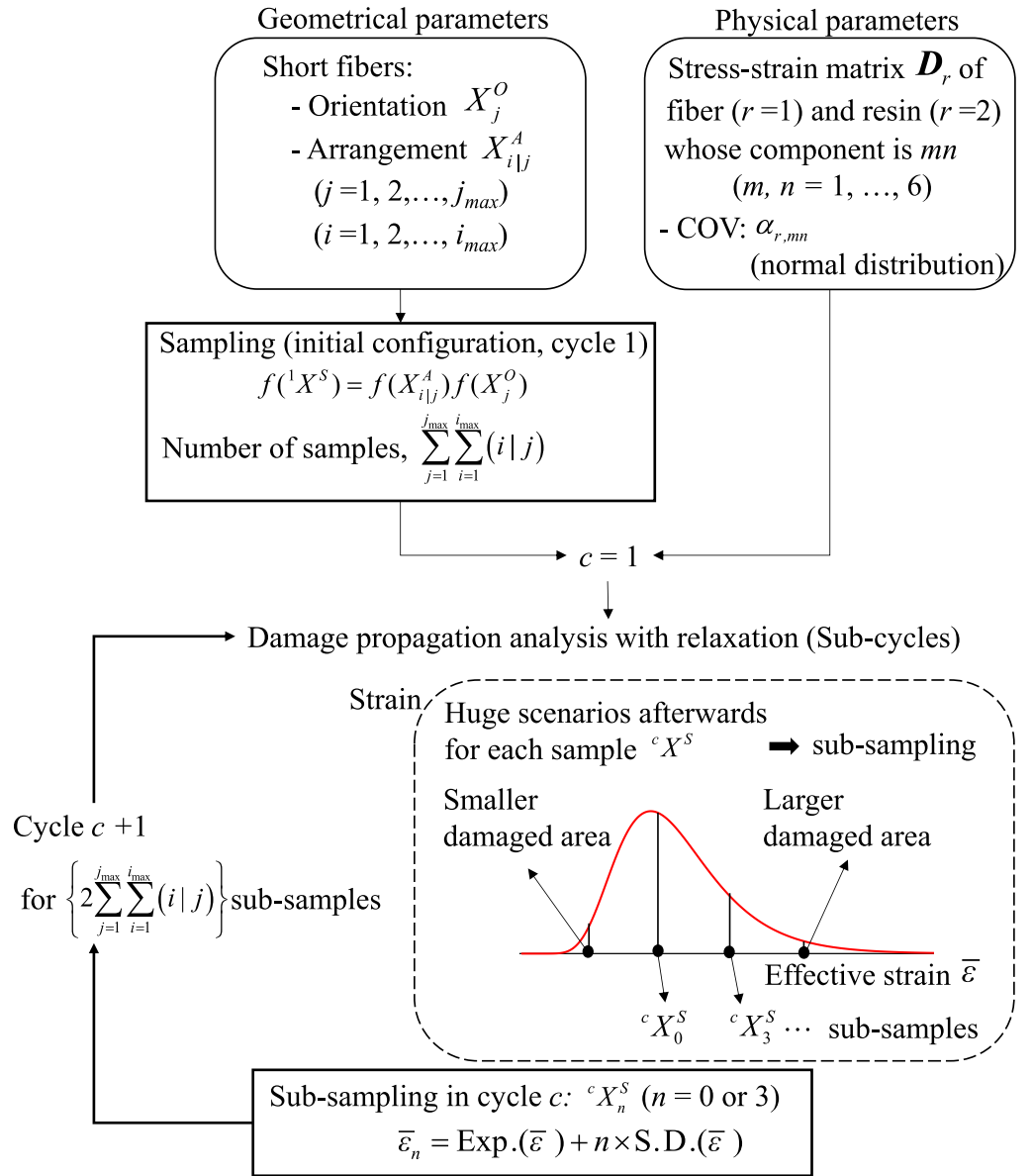

(a) Sampling and sub-sampling

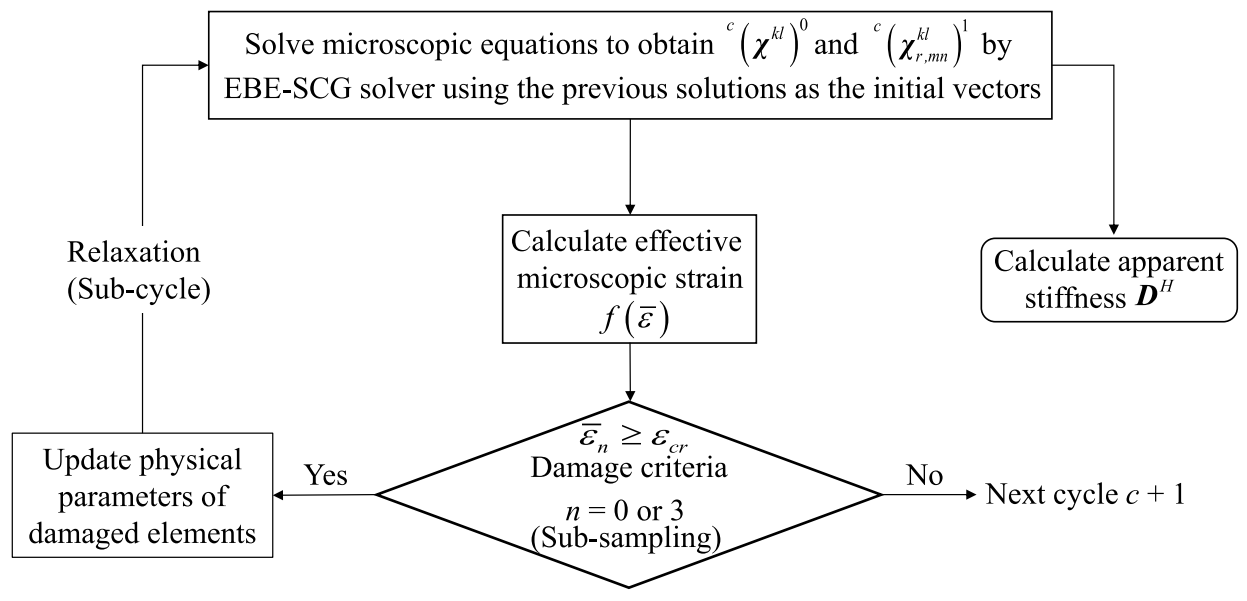

(b) Cycle and sub-cycle

\subsection{Nonlinear computational scheme considering microscopic stochastic damage propagation}

In this section, a nonlinear computational scheme of stochastic microscopic damage propagation considering both physical and geometrical randomness is presented. The random physical parameters $\alpha_{r, m n}$ are considered theoretically in FPSH, but sampling is used for random geometrical parameters $X_{j}^{O}$ and $X_{i \mid j}^{A}$ at the initial state of the first cycle $c=1$. When damage appears under applied strain conditions, and then the scenario becomes huge afterward for each sample ${ }^{c} X^{5}$ in the stochastic nonlinear analysis, sub-sampling ${ }^{C} X_{n}^{S}$ is applied (see Fig. 5a). Where $n$ 
is the level of the standard deviations. In this work, two sub-samplings ${ }^{C} X_{0}^{S}$ and ${ }^{C} X_{3}^{S}$ corresponding to the expected value ( $50 \%$ of probability) and three deviation level $(99.7 \%$ of probability) are addressed.

Many popular damage criteria for the damage model, such as maximum stress, maximum strain [13] as well as Tsai-Hill, Tsai-Wu [10, 11], Hoffman [37], Hashin [38], were widely used. In this framework, an arbitrary failure rule is accepted, but just for simplicity, a non-interactive criterion using maximum effective strain $\varepsilon_{c r}$ is employed. This criterion considers that the element of the matrix phase fails when its Mises microscopic strain exceeds the respective allowable $\varepsilon_{c r}$ (see Fig. 5b). This criterion can be extended to reliability analysis when the respective allowable is considered as a random variable $f\left(\varepsilon_{c r}\right)$. Based on the results of characteristic displacements solved by the EBE-SCG solver in FPSH, effective microscopic strains are calculated in the different levels of standard deviations. Once an element is damaged, the mechanical properties of that element will be updated by damaged properties which result in the stiffness degradation. Following this, the characteristic displacements must be simultaneously updated in the lefthand side loop, so-called the sub-cycle (see Fig. 5b). The calculations are performed continuously under the same macroscopic strain level until there is no element damaged anymore. During this sub-cycle computation, the strain relaxation is achieved. If damage does not occur, the macroscopic strain increases with small increments and afterward, the iterative process repeats according to the updated microscopic strain at the next cycle $c+1$. The simulation of each model is stopped until there is no damage to occur and no given macroscopic strain to update.

\section{Numerical example}

\subsection{Sampling}

For illustration in this work, 11 SVE models, containing 16 short glass fibers with 3 fiber lengths of $0.435 \mathrm{~mm}, 0.543 \mathrm{~mm}$, and $0.645 \mathrm{~mm}$ in yellow, green, red color respectively, are demonstrated for the proposed stochastic nonlinear damage scheme as shown in Fig. 6. The matrix is transparent. The size of the SVE is $224 \mathrm{~mm} \times 224 \mathrm{~mm} \times 672 \mathrm{~mm}$ suitable to model the skin layer and core layer as well. The volume fraction of fiber is

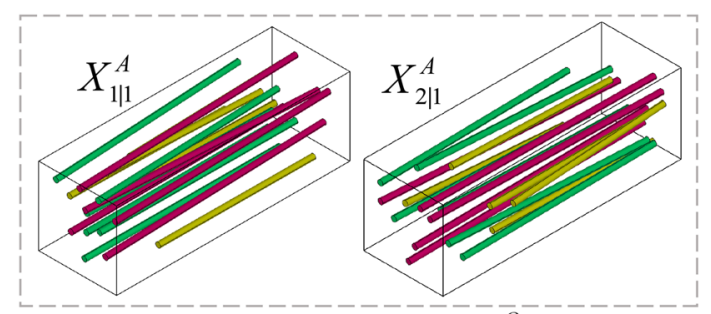

(a) Fiber orientation $X_{1}^{O}$

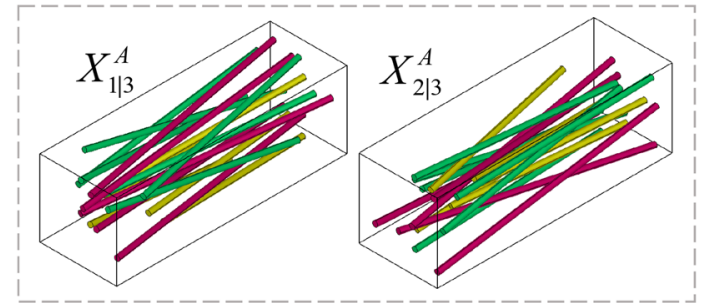

(c) Fiber orientation $X_{3}^{O}$

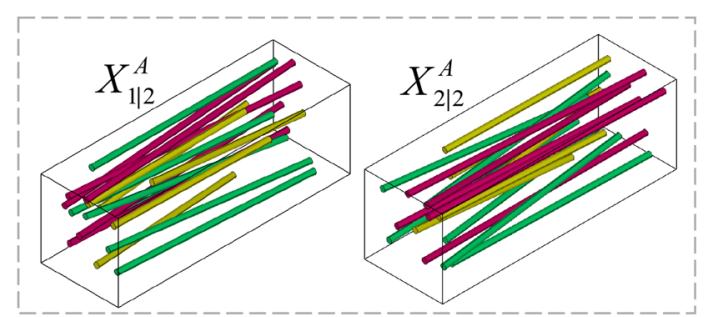

(b) Fiber orientation $X_{2}^{O}$

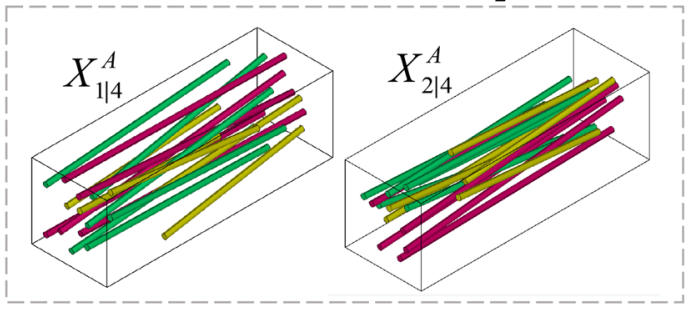

(d) Fiber orientation $X_{4}^{O}$

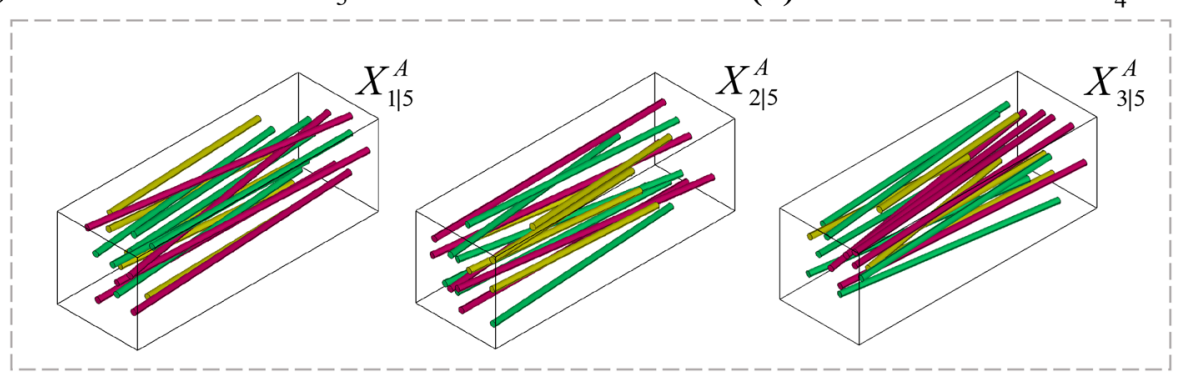

(e) Fiber orientation $X_{5}^{O}$

Fig. 6 Initial configuration of samples for cycle $1,{ }^{1} X^{S}$ 
Table 1 Setting of samplings

\begin{tabular}{lllll}
\hline $\begin{array}{l}\text { Orientation } \\
\text { sample No. }\end{array}$ & $\begin{array}{l}\text { Fiber } \\
\text { orienta- } \\
\text { tion } X_{j}^{O}\end{array}$ & Angle $\varphi$ & Angle $\theta$ & $\begin{array}{l}\text { Number of samples } \\
\text { w.r.t fiber arrange- } \\
\text { ment, } i_{\max }\end{array}$ \\
\hline 1 & $X_{1}^{O}$ & {$[-3,3]$} & {$[87,93]$} & 2 \\
2 & $X_{2}^{O}$ & {$[-6,6]$} & {$[84,96]$} & 2 \\
3 & $X_{3}^{O}$ & {$[-10,10]$} & {$[80,100]$} & 2 \\
4 & $X_{4}^{O}$ & {$[-3,3]$} & {$[80,100]$} & 2 \\
5 & $X_{5}^{O}$ & {$[-10,10]$} & {$[87,93]$} & 3 \\
\hline
\end{tabular}

Table 2 Properties of constituent materials

\begin{tabular}{lllll}
\hline & $\begin{array}{l}\text { Material } \\
\text { number, } r\end{array}$ & $\begin{array}{l}\text { Young's } \\
\text { modulus } \\
(\mathrm{GPa})\end{array}$ & Poisson's ratio & $\begin{array}{l}\text { Random } \\
\text { parameter } \\
\alpha_{r, m n}\end{array}$ \\
\hline Fiber & 1 & 72 & 0.26 & - \\
Matrix & 2 & 3.79 & 0.37 & 0.03 \\
\hline
\end{tabular}

4.5\%. We seek to investigate the influences of fiber orientation and fiber arrangement on the damage propagation of the injection molded short fiber reinforced plastic in the angel range $\left[-10^{0}, 10^{\circ}\right]$ of fiber orientation. All input parameters of the models generated by Meshman Particle Packing are provided in Table 1. There are 5 random fiber orientation samples with respect to its random fiber arrangements. The properties of constituent materials are given in Table 2 [24]. The properties of fibers and the matrix are employed corresponding to E-glass and Epoxy resin. Only the damage of the resin is performed in this example. The randomness of the material can be considered separately for each component of the stress-strain matrix of each constituent. In this example, to investigate the influence of only random physical parameter in the matrix phase and due to the limitation of the first order perturbation expansion, the randomness of this material is set in the same way as in our previous papers [13]. Once the elements of the matrix phase are damaged, the random physical parameters of these elements are set to be zero.

\subsection{Results}

Many probable damage patterns of the short glass fiber reinforced composite are predicted and visualized in the three-dimension from the stochastic nonlinear simulation when considering randomness in both physical and geometrical parameters. In this simulation, a combined

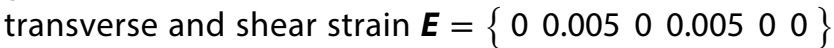
was assumed for the applied macroscopic strain. For short expression, we used $\boldsymbol{E}=0.005$ instead of the above one.
The damage criterion utilizing for the effective strain in the matrix phase is assumed as $\varepsilon_{c r}=0.011$. The initial damages of the models were detected at $\boldsymbol{E}=0.003$. The results were verified by trying other smaller macroscopic strain increments with no significant difference. The contact between fiber and matrix is considered perfect. The number of 10-noded tetrahedral elements of 11 SVE models goes from 318,093 to 332,027 with more than 4 hundreds of thousands of degrees of freedom. Damage initiation refers to the beginning of the degradation of the stiffness of finite matrix elements. The process of degradation begins when the microscopic effective strain satisfies the damage criterion. Since the value of the effective microscopic strain in the matrix element exceeds the strain threshold, the properties of this element are changed, and Young's modulus of this element is set to a very low value about $0.00,001 \%$ of the initial value in this work. The numbers of failed elements are printed out in a file, which was used to visualize the calculated damage evolution in the matrix phase.

Some damage patterns of the material models without (top) and with (bottom) transparent boxes are shown in Fig. 7. Damaged resin appears near the fibers and then penetrates to other neighbor fibers. As the random fiber orientation and fiber arrangement, the damage patterns grow complexly in the different modes. Using the proposed scheme, the damages of the random microstructures can be captured even though the morphologies are quite complex. Additionally, even with the same morphology of the same model, this stochastic simulation can capture different damage patterns with different damaged volume fractions when considering quite small random physical parameter in the matrix phase. For illustration, the results of two other models $X_{1 \mid 2}^{A}$ and $X_{1 \mid 4}^{A}$ are shown in Fig. 8. With the same morphology of the model $X_{1 \mid 2^{\prime}}^{A}$ the observed stochastic damaged volume fraction corresponding to the three standard deviation level is $4.45 \%$ larger than the observed deterministic one corresponding to $50 \%$ of probability. It can be concluded that the variability of the microstructure has a significant effect on the damage growth. The proposed scheme is one step to develop the virtual damage testing tool for complex microstructures at the microscale.

In this part, the microscopic strain distributions resulted from the above stochastic damage simulation are discussed. The significant influences of the variability in the physical and geometrical parameters on the microscopic strains are figured out as shown in Fig. 9. The strain distributions in the matrix are highlighted on the high strain values and the differences among the models. The average strain is obtained by using Eq. (3). It can be observed that the variation of the microscopic strains on the right 

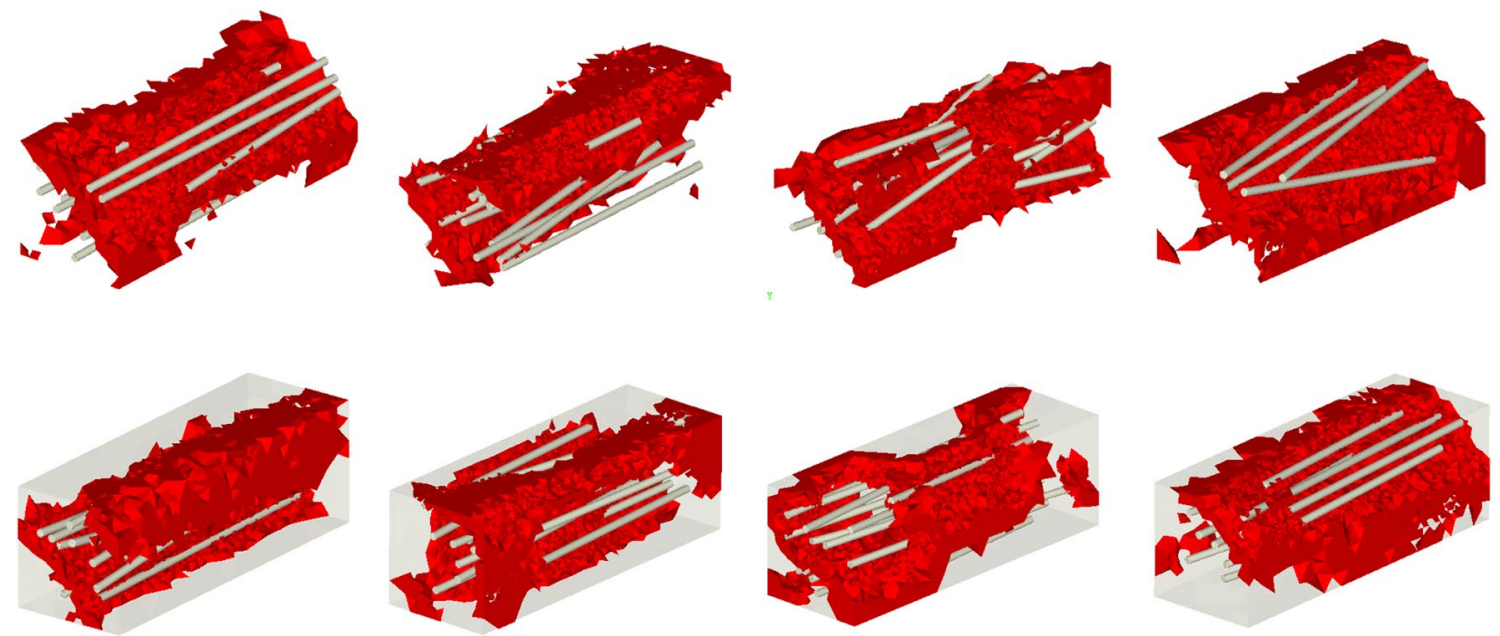

Fig. 7 Some damage patterns predicted at cycle $3,{ }^{3} X_{n}^{5}(n=0$ or 3$)$
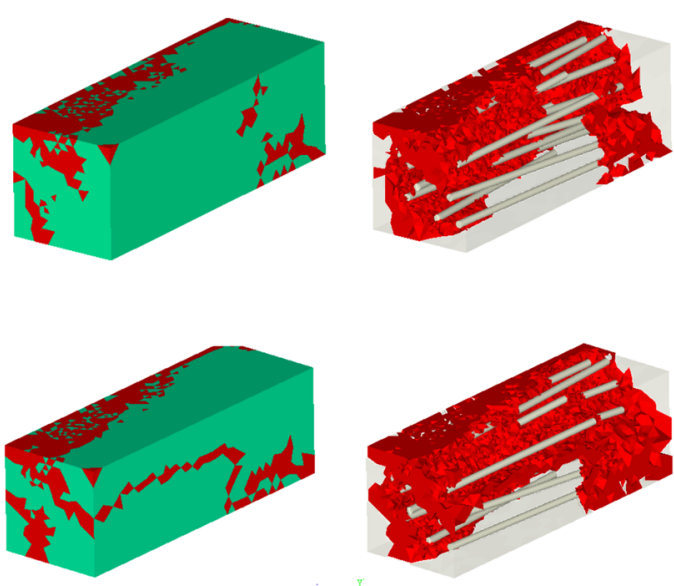

(a) Model $X_{1 \mid 2}^{A}$

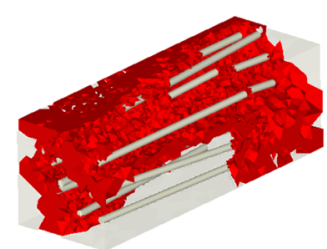

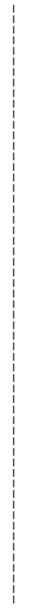
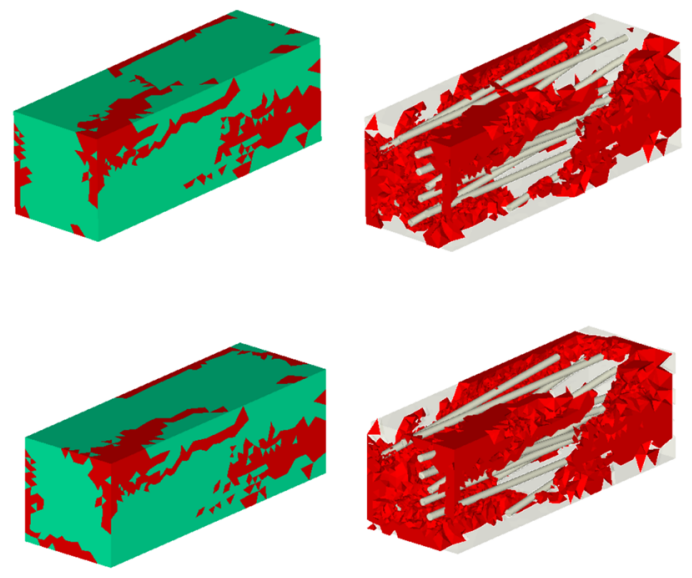

(b) Model $X_{114}^{A}$

Fig. 8 Influence of physical parameters on damage pattern when the properties of matrix are (top) average or (bottom) 3 times of standard deviation at cycle $3,{ }^{3} X_{n}^{S}[n=0$ (top) or 3 (bottom)]

distribution tail which expresses the number of large strain values is more significant than that of the left tail which expresses the number of small strain values in case of linear analysis due to the macroscopic strain $\boldsymbol{E}=0.003$ as shown in Fig. 9a. It means that a huge scenario of the damage propagations is considered at the initial stage before the damage evolution based on the stochastic calculation of microscopic strains. The variability among the strain distributions is much complex since the damage propagates during the stochastic damage process.
Figure 10 shows the probable degradation of the apparent stiffness (i.e., $D_{11}^{H}, D_{22}^{H}$ and $D_{44}^{H}$ ) in the range of the threestandard deviation during the damage propagation. It can be seen from $D_{11}^{H}$ that the composite is stiffer in the direction of alignment of the fibers when compared to the other directions. Initially isotropic constituent materials change the composite properties to orthotropic due to the applied macroscopic strain. At the final damage state, the degradation of $D_{22}^{H}$ was faster than those of other components due to the applied strain conditions, 


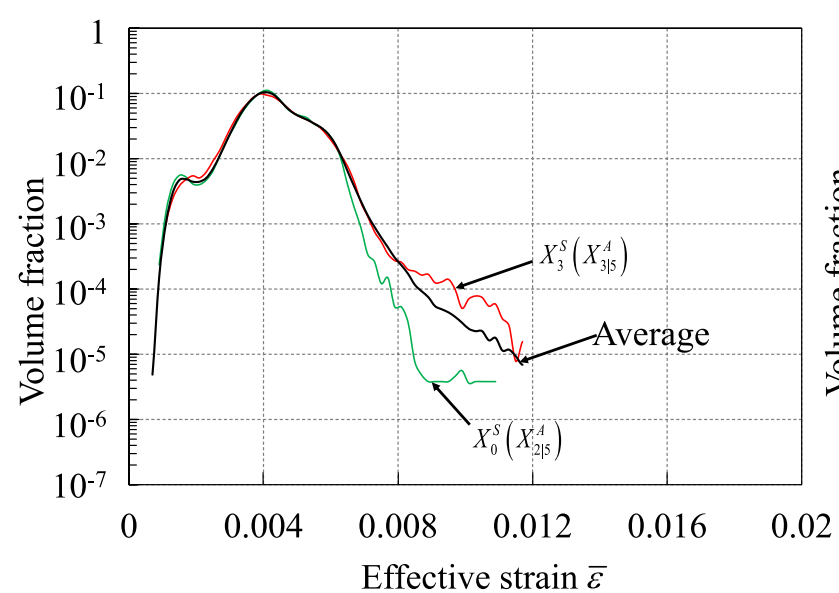

(a) Linear analysis before damage occurred

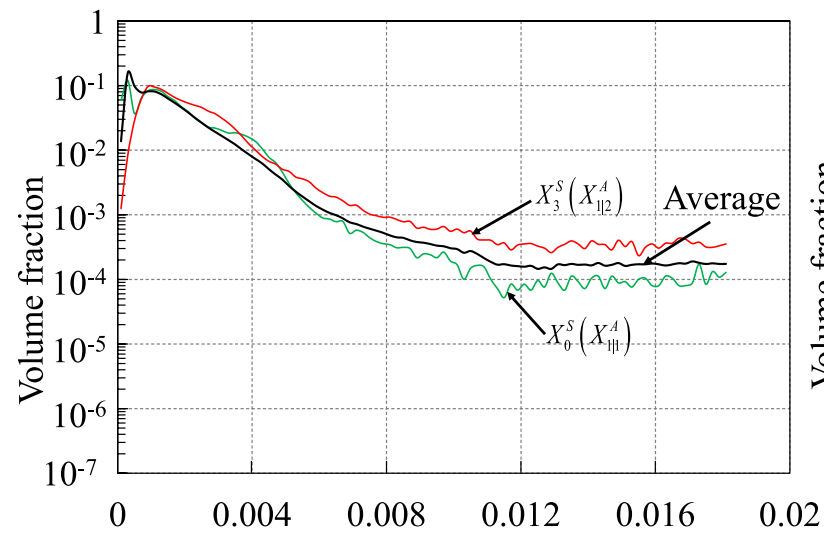

Effective strain $\bar{\varepsilon}$

(c) Applied macroscopic strain $\mathbf{E}=0.004$

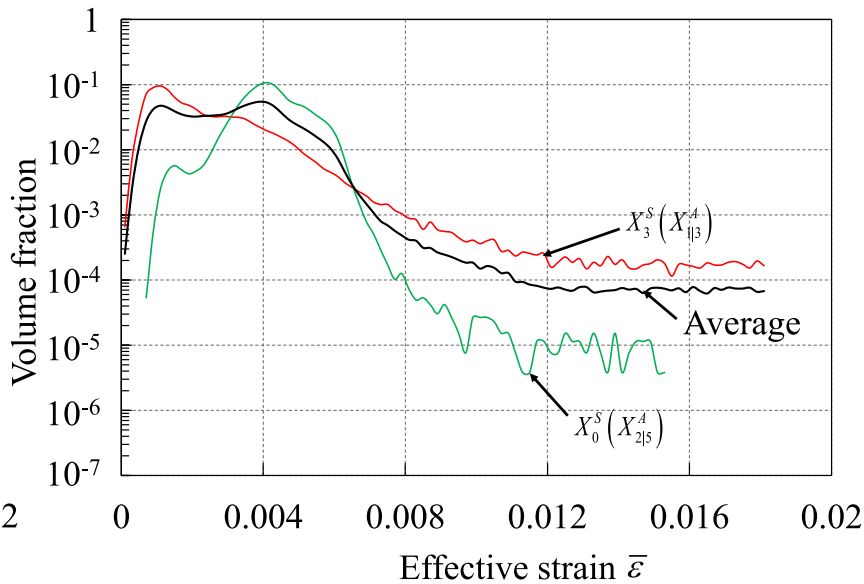

(b) Applied macroscopic strain $\mathbf{E}=0.003$

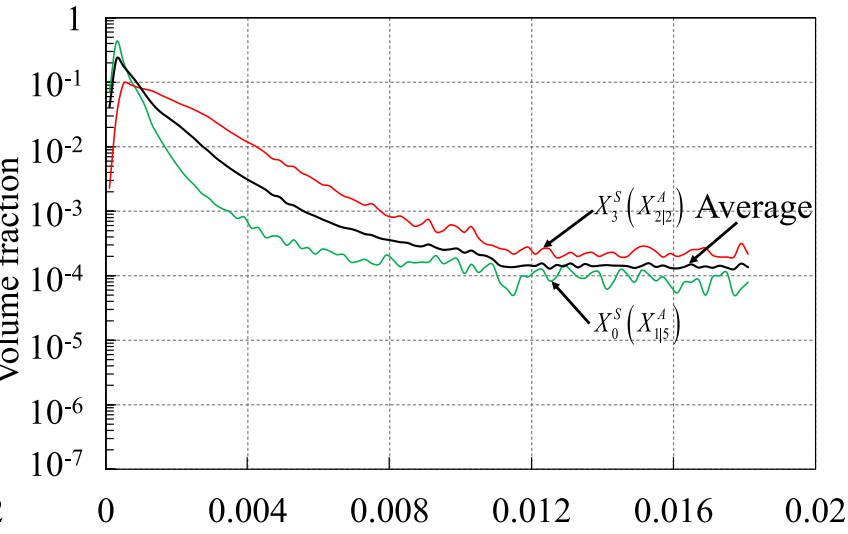

Effective strain $\bar{\varepsilon}$

(d) Applied macroscopic strain $\mathbf{E}=0.005$

Fig. 9 Strain distributions in matrix highlighting on high strain value and on the differences among models

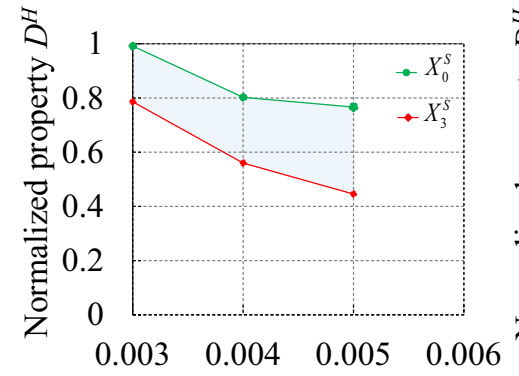

Macroscopic strain $\boldsymbol{E}$

(a) $D_{11}^{H}$

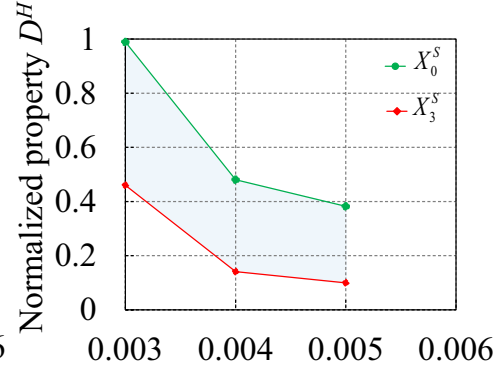

Macroscopic strain $\boldsymbol{E}$

(b) $D_{22}^{H}$

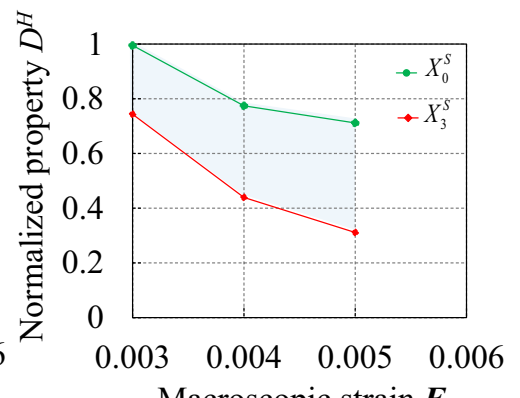

Macroscopic strain $\boldsymbol{E}$

(c) $D_{44}^{H}$

Fig. 10 Degradation of apparent stiffness

and the material was getting weakest in transverse directions since the components $D_{22}^{H}$ is reduced catastrophically. The light solid color shows the region of the degradation of the apparent stiffness considering the variability in the physical and geometrical parameters in the nonlinear stochastic simulation. 


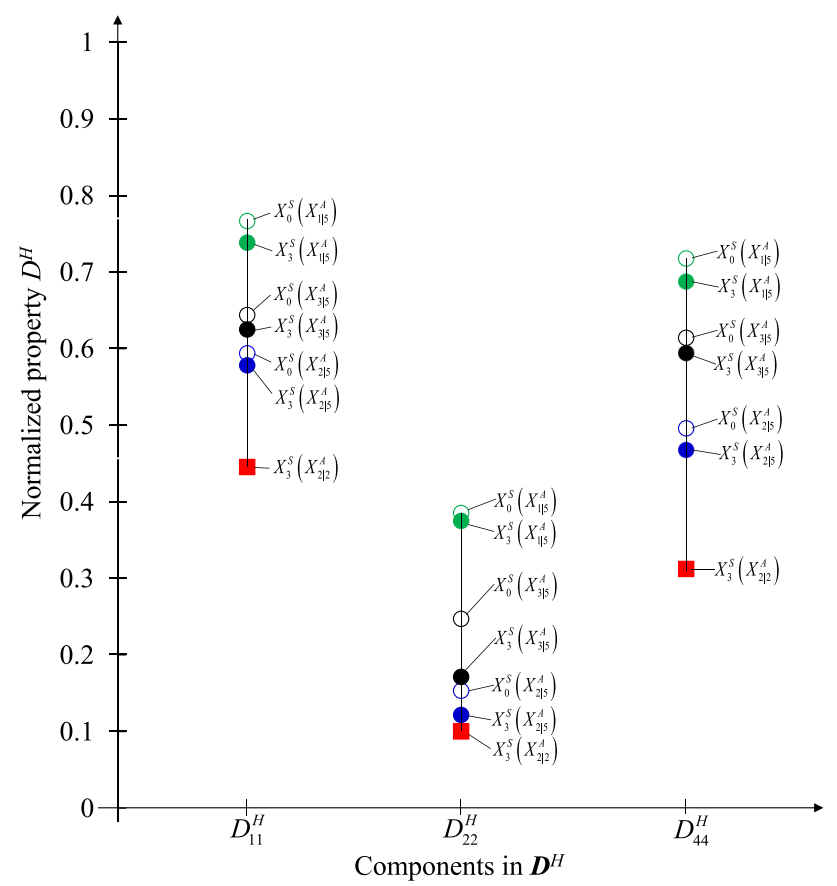

Fig. 11 SVE specification in degraded apparent stiffness with enlarged views on the components $D_{11^{\prime}}^{H} D_{22^{\prime}}^{H} D_{44}^{H}$ due to $\mathbf{E}=0.005$ (Only the results of orientation samples 2 and 5 are shown)

\subsection{Discussion}

As shown in Fig. 9a, at the initial state before the damage occurred, the higher effective strain distribution was found in case of $X_{3}^{S}\left(X_{3 \mid 5}^{A}\right)$ and the minimum one was found in case of $X_{0}^{S}\left(X_{2 \mid 5}^{A}\right)$. This tells that the influence of the arrangement in the same orientation sample gave the maximum and minimum effective strain distributions and therefore the influence of the arrangement can lead to conclude that it is more significant than the influence of the orientation in this analysis. At cycle 3 with $\boldsymbol{E}=0.005$ as shown in Fig. 9d, it is quite reasonable that the maximum effective strain distribution was founded for $X_{3}^{S}\left(X_{2 \mid 2}^{A}\right)$ and the minimum one is $X_{0}^{S}\left(X_{1 \mid 5}^{A}\right)$, which respectively agreed with the minimum and maximum of the degraded stiffness of the components $D_{11^{\prime}}^{H} D_{22^{\prime}}^{H} D_{44}^{H}$ as shown in Fig. 11, where the results of only some samplings were visible.

In the case of the orientation sample 5 with 3 different arrangements, by comparing the degraded stiffness among 3 SVE models, i.e. $X_{0}^{S}\left(X_{1 \mid 5}^{A}\right), X_{0}^{S}\left(X_{2 \mid 5}^{A}\right), X_{0}^{S}\left(X_{3 \mid 5}^{A}\right)$ (blank circles in Fig. 11) of $D_{11^{\prime}}^{H} D_{22^{\prime}}^{H} D_{44^{\prime}}^{H}$ it can be pointed out that large difference was found among them. Additionally, the damaged volume evolution of the different arrangements for the same orientation samples resulted in quite different behavior. For instance, only the results of the orientation samples 2 and 5 are shown in Fig. 12 .
Fig. 12 Influence of fiber arrangement on damaged volume evolution

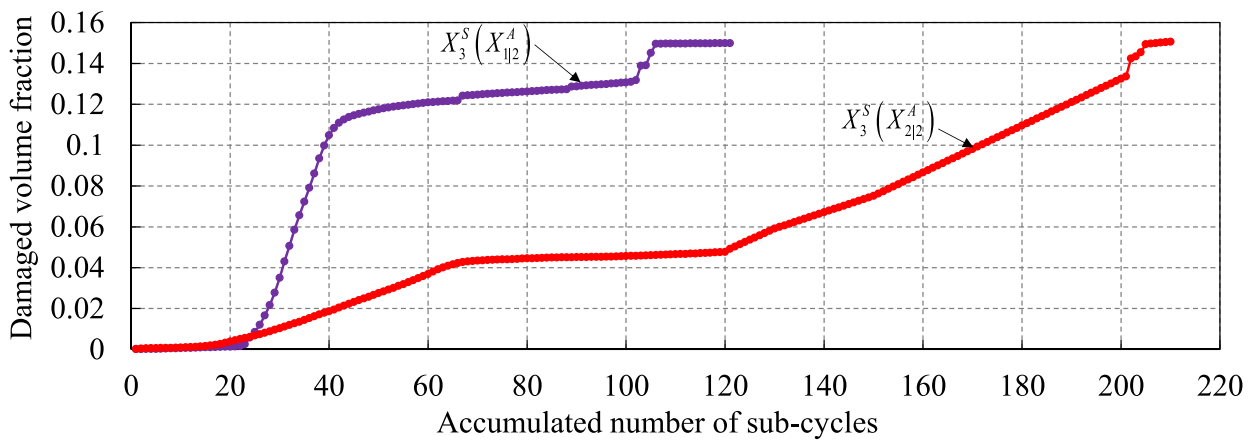

(a) Two models of orientation sample No. 2, $X_{2}^{O}$

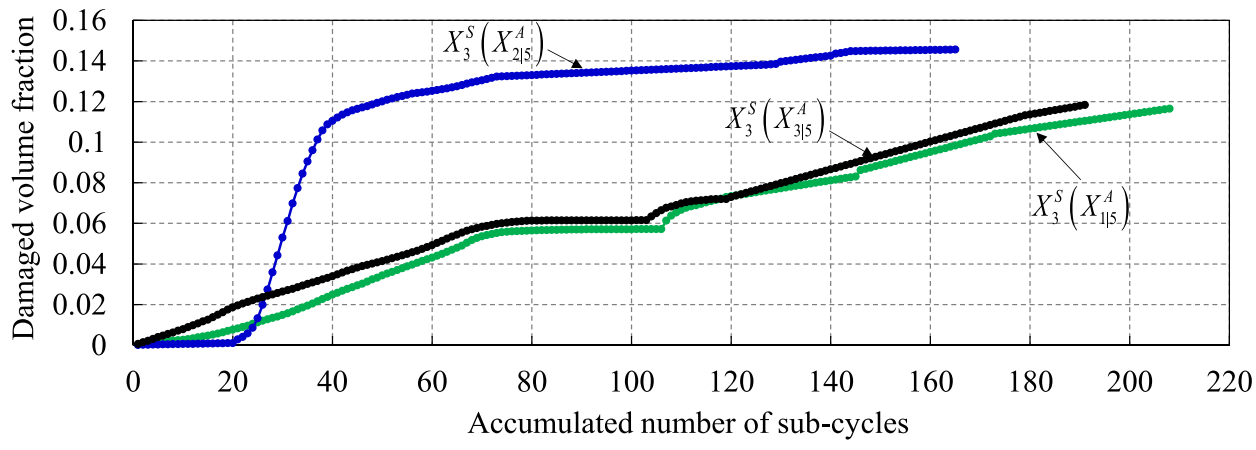

(b) Three models of orientation sample No. $5, X_{5}^{O}$ 


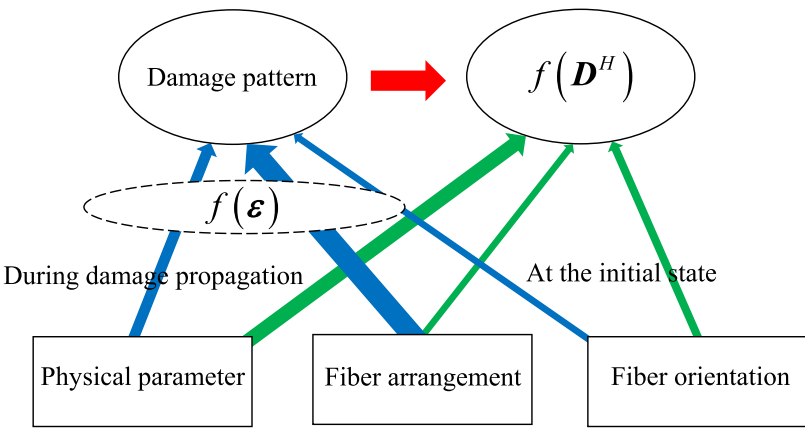

Fig. 13 Variability influence level of physical and geometrical parameters
Furthermore, looking at the results of sub-samplings $X_{0}^{S}$ and $X_{3}^{S}$ (blank and solid circles) for the 3 SVEs of the orientation sample 5 in Fig. 11, the variability influence of the physical parameters on the degraded stiffness was smaller in most of the cases than that of the arrangement or orientation. To this end, we can illustrate the influential level (blue arrows) of the physical and geometrical parameters on the strain distributions and the homogenized stiffness as shown in Fig. 13.

However, the results in the linear analysis of the probabilistic density function of $\boldsymbol{D}_{r, m n}^{H}$ pointed out that the variability influence of the physical parameter is larger than the variability influence of geometrical parameters (see Fig. 14). The mixture distribution of the homogenized properties was obtained by Eq. (2). In this state, the

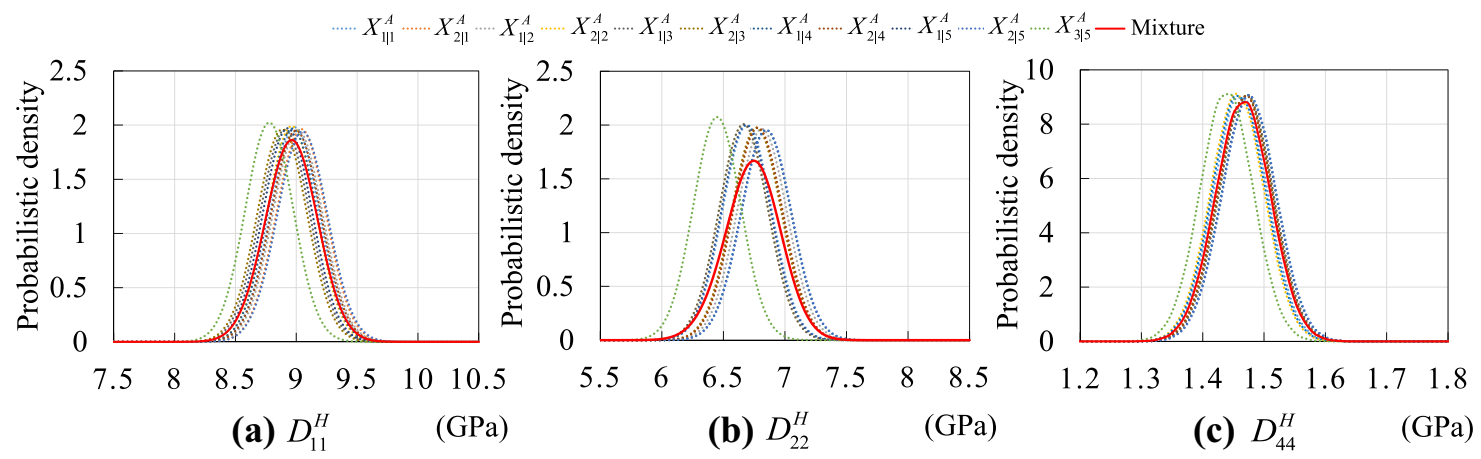

Fig. 14 Probabilistic density function of $D^{H}$ in the linear analysis

Fig. 15 Number of EBE-SCG iterations and damaged volume evolution of a sample $X_{3}^{S}\left(X_{1 \mid 2}^{A}\right)$

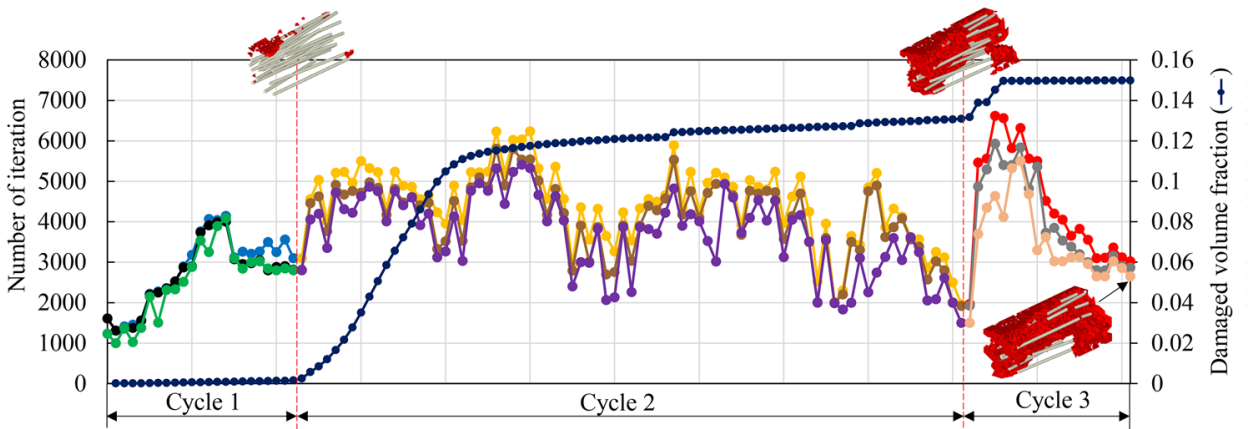

(a) Solution of zeroth order terms of characteristic displacements

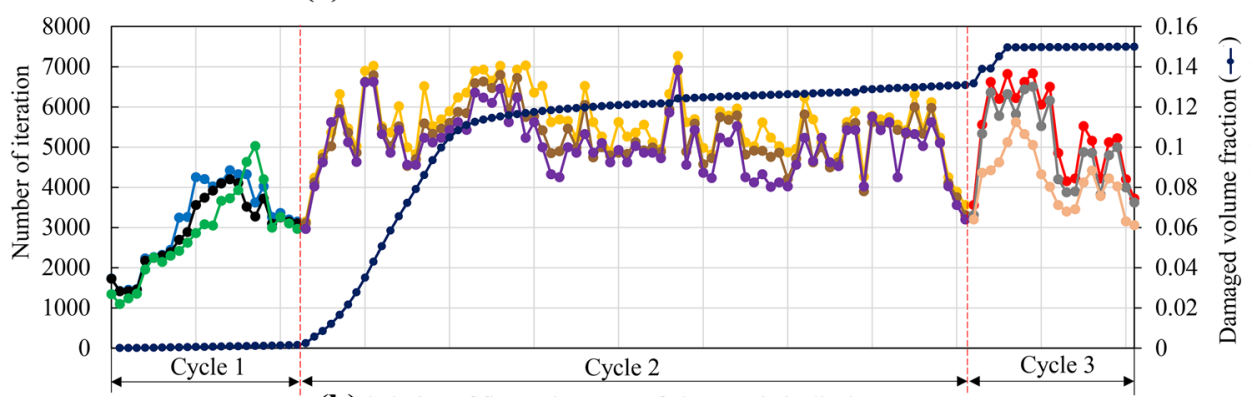

(b) Solution of first order terms of characteristic displacements 
Fig. 16 Number of EBE-SCG iterations and damaged volume evolution of a sample $X_{3}^{S}\left(X_{1 \mid 4}^{A}\right)$

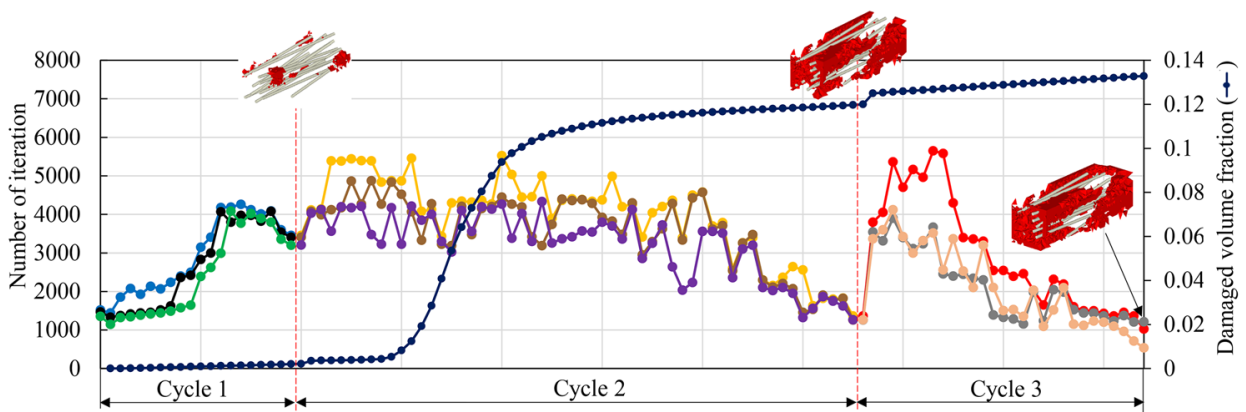

(a) Solution of zeroth order terms of characteristic displacements

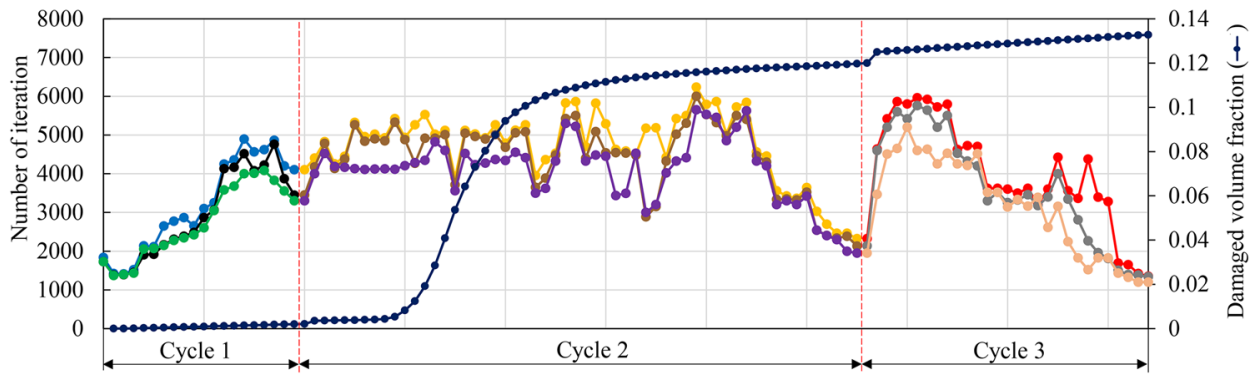

(b) Solution of first order terms of characteristic displacements
Table 3 Legend of iterations

\begin{tabular}{cccc}
\hline & \multicolumn{3}{c}{ Number of cycle } \\
\hline & 1 & 2 & 3 \\
\hline$\left(\chi^{11}\right)^{0}$ & $\rightarrow$ & $\rightarrow$ & $\rightarrow$ \\
$\left(\chi^{22}\right)^{0} \rightarrow \rightarrow$ & $\rightarrow-$ & $\rightarrow$ \\
$\left(\chi^{23}\right)^{0}$ & $\rightarrow$ & $\rightarrow$ & $-\rightarrow$ \\
\hline
\end{tabular}

influence level (green arrows) of the physical and geometrical parameters can be shown in Fig. 13.

The acceleration by using the previous solutions as initial vectors has already been shown in our previous paper [13]. Figures 15 and 16 with Table 3 show the number of the EBE-SCG iterations and damaged volume evolutions of two samples $X_{3}^{S}\left(X_{1 \mid 2}^{A}\right)$ and $X_{3}^{S}\left(X_{1 \mid 4}^{A}\right)$. For the solutions of the zeroth-order and the first-order terms of the characteristic displacements of both samples, when the damaged volume increased significantly, the number of iterations did not always increase. The reason is that the previous solutions worked well for the faster convergence even though the damaged volume increased. As the increase of sub-cycle numbers, the damaged volume is saturated and together with that, the number of SCG iteration is decreased. But at the beginning of the cycle, the macroscopic strain is increased, so the number of iteration was again larger.

\section{Concluding remarks}

In this paper, a stochastic multiscale computational scheme for the damage propagation considering the physical and geometrical variabilities in the microstructures of short glass fiber reinforced composites was performed. The FPSH method was used to take into account the variability in the physical parameters of the resin, whereas the sampling scheme was used for the variability of the geometrical parameters (fiber orientation, fiber arrangement) of the material. The sub-sampling was used in the stochastic nonlinear damage simulation since the scenarios become huge. The sub-sampling is very useful when the variability of the damage threshold is considered, although it is assumed to be deterministic in this paper. A huge number of the probable damage patterns of the short fiber reinforced plastic were predicted and visualized in the three-dimension to show many scenarios of damage propagations. The stochastic simulation can capture the different damage patterns of the same morphology in one model even considering quite small random physical parameter in the matrix phase. The influential level of the variability in the microstructure of the material on microscopic strains, damage evolutions, and degraded properties were discussed. Even though the results in the linear analysis of the homogenized properties of the material pointed out that the influence of the physical parameter is larger than the influence of geometrical parameters, the influence of the fiber arrangement is more significant than the influences of the physical parameter and the fiber orientation in a small range 
during the damage evolution. The stochastic simulation is useful for the quality assurance of composite materials and structures when the experimental results have large variability and have to be collected by doing big enough times. In further research, the proposed scheme can be applied to consider the uncertainty or variability in the constraint condition and loading condition, where the concept of the sub-sampling may work when the scenario gets huge. Additionally, uncertainty or variability quantification in nonlinear multiscale computational simulation should be further investigated.

However, the present study has some limitations and is open to several improvements. For instance, the proposed stochastic method should also consider the interface and interphase damage behavior, fiber failure as well as matrix failure with different practical damage models. The homogenization method should not be used once the localization occurs. One of the popular methods proposed by Rukavina et al. [39], which can consider a fully damage process including a complete set of failure modes, should be considered in further work.

Acknowledgements This work was supported by a Japan Society for the Promotion of Science Grant-in-Aid for Scientific Research (B) (KAKENHI Grant No. 16H04239). The first author thanks the Ministry of Education, Culture, Sports, Science and Technology of Japan for support in the form of a full scholarship to study and research at Keio University.

\section{Compliance with ethical standards}

Conflict of interest The authors declare that they have no conflict of interest.

\section{References}

1. Chen NZ, Guedes Soares C (2008) Spectral stochastic finite element analysis for laminated composite plates. Comput Methods Appl Mech Eng 197(51):4830-4839

2. Sepahvand K (2016) Spectral stochastic finite element vibration analysis of fiber-reinforced composites with random fiber orientation. Compos Struct 145:119-128

3. Sasikumar P, Venketeswaran A, Suresh R, Gupta S (2015) A data driven polynomial chaos based approach for stochastic analysis of CFRP laminated composite plates. Compos Struct $125 \cdot 212-227$

4. Kamin M (2007) Generalized perturbation-based stochastic finite element method in elastostatics. Comput Struct 85(10):586-594

5. Basaruddin KS, Takano N, Akiyama H, Nakano T (2013) Uncertainty modeling in the prediction of effective mechanical properties using stochastic homogenization method with application to porous trabecular bone. Mater Trans 54:1250-1256

6. Talha M, Singh BN (2014) Stochastic perturbation-based finite element for buckling statistics of FGM plates with uncertain material properties in thermal environments. Compos Struct 108:823-833
7. Zhou XY, Gosling PD, Pearce CJ, Ullah Z, Kaczmarczyk L (2016) Perturbation-based stochastic multi-scale computational homogenization method for woven textile composites. Int J Solids Struct 80:368-380

8. Wen P, Takano N, Kurita D (2016) Probabilistic multiscale analysis of three-phase composite material considering uncertainties in both physical and geometrical parameters at microscale. Acta Mech 227(10):2735-2747

9. Wen P, Takano N, Akimoto S (2018) General formulation of the first-order perturbation based stochastic homogenization method using many random physical parameters for multiphase composite materials. Acta Mech 229(5):2133-2147

10. Dong JW, Feng ML (2010) Asymptotic expansion homogenization for simulating progressive damage of 3D braided composites. Compos Struct 92(4):873-882

11. Zhou XY, Gosling PD, Ullah Z, Kaczmarczyk L, Pearce CJ (2017) Stochastic multi-scale finite element based reliability analysis for laminated composite structures. Appl Math Model 45:457-473

12. Ramírez-Torres $A$, Penta R, Rodríguez-Ramos R, Merodio J, Sabina FJ, Bravo-Castillero J, Guinovart-Díaz R, Preziosi L, Grillo A (2018) Three scales asymptotic homogenization and its application to layered hierarchical hard tissues. Int J Solids Struct 130:190-198

13. Hoang TD, Takano N (2019) First-order perturbation-based stochastic homogenization method applied to microscopic damage prediction for composite materials. Acta Mech 230(3):1061-1076

14. Harris CE, Starnes JH, Shuart MJ (2001) An assessment of the state-of-the-art in the design and manufacturing of large composite structures for aerospace vehicles. NASA TM 210844

15. Feraboli P, Deleo F, Wade Rassaian B, Higgins M, Byar A (2010) Predictive modeling of an energy-absorbing sandwich structural concept using the building block approach. Compos Part A 41(6):774-786

16. Kubo G, Matsuda T (2016) Effects of laminate misalignment on macroscopic strength and microscopic damage development of plain-woven laminates. Mech Eng Lett 2:16-00248

17. Hagiwara K, Ishijima S, Takano N, Otani A, Nakai A (2017) Parameterization, statistical measurement and numerical modeling of fluctuated meso/micro-structure of plain woven fabric GFRP laminate for quantification of geometrical variability. Mech Eng J 4(4):17-00053

18. Takano N, Asai M, Okamoto K (2012) Monte carlo simulation of dynamic problem using model order reduction technique highlighting on tail probability. J Comput Sci Technol 6(3):169-181

19. Mortazavian S, Fatemi A (2015) Effects of fiber orientation and anisotropy on tensile strength and elastic modulus of short fiber reinforced polymer composites. Compos Part B 72:116-129

20. Phelps JH, Abd El-Rahman Al, Kunc V, Tucker CL (2013) A model for fiber length attrition in injection-molded long-fiber composites. Compos Part A 51:11-21

21. Ioannis I, Alma H, Inna G, loannis I, Alma H, Inna G, Costas S, Almaadeed MA (2014) Micro-mechanical parameters in short fibre composite. Appl Compos Mater 21(1):197-211

22. Ogierman W, Kokot $\mathrm{G}$ (2016) A study on fiber orientation influence on the mechanical response of a short fiber composite structure. Acta Mech 227(1):173-183

23. Chen CS, Chen TJ, Chen SC, Chien RD (2011) Optimization of the injection molding process for short-fiber-reinforced composites. Mech Compos Mater 47(3):359-368

24. JrL Mishnaevsky, Brøndsted P (2009) Micromechanisms of damage in unidirectional fiber reinforced composites: 3D computational analysis. Compos Sci Technol 69(7-8):1036-1044

25. Sasayama T, Okabe T, Aoyagi Y, Nishikawa M (2013) Prediction of failure properties of injection-molded short glass fiber-reinforced polyamide 6,6. Compos Part A 52:45-54 
26. Notta-Cuvier D, Lauro F, Bennani B, Balieu R (2014) Mechanics of materials damage of short-fibre reinforced materials with anisotropy induced by complex fibres orientations. Mech Mater 68:193-206

27. Wang HW, Zhou HW, Ji HW, Zhang XC (2014) Application of extended finite element method in damage progress simulation of fiber reinforced composites. Mater Des 55:191-196

28. Jha NK, Reinoso J, Dehghani H, Merodio J (2019) A computational model for fiber-reinforced composites: hyperelastic constitutive formulation including residual stresses and damage. Comput Mech 63(5):931-948

29. Hun DA, Guilleminot J, Yvonnet J, Bornert M (2019) Stochastic multiscale modeling of crack propagation in random heterogeneous media. Int J Numer Methods Eng 119(13):1325-1344

30. Matouš K, Geers MG, Kouznetsova VG, Gillman A (2017) A review of predictive nonlinear theories for multiscale modeling of heterogeneous materials. J Comput Phys 330:192-220

31. Lutz W, Herrmann J, Kockelmann M, Hosseini HS, Jäckel A, Schmauder S, Busse G (2009) Damage development in shortfiber reinforced injection molded composites. Comput Mater Sci 45(3):698-708

32. Selmi A, Doghri I, Adam L (2011) Micromechanical simulations of biaxial yield, hardening and plastic flow in short glass fiber reinforced polyamide. Int J Mech Sci 53(9):696-706

33. Meshman_ParticlePackingVer.2.2. http://www.meshman.jp/ meshman/particlepacking.html. Accessed 10 Sept 2019
34. Bogdanor MJ, Oskay C, Clay SB (2015) Multiscale modeling of failure in composites under model parameter uncertainty. Comput Mech 56(3):389-404

35. Guedes J, Kikuchi N (1990) Preprocessing and postprocessing for materials based on the homogenization method with adaptive finite element methods. Comput Methods Appl Mech Eng 83(2):143-198

36. Takano N, Ohnishi Y, Zako M, Nishiyabu K (2000) The formulation of homogenization method applied to large deformation problem for composite materials. Int J Solids Struct 37(44):6517-6535

37. Takano N, Uetsuji Y, Kashiwagi Y, Zako M (1999) Hierarchical modelling of textile composite materials and structures by the homogenization method. Model Simul Mater Sci Eng 7(2):207-231

38. Zhang C, Curiel-Sosa JL, Bui TQ (2019) Meso-scale finite element analysis of mechanical behavior of 3D braided composites subjected to biaxial tension loadings. Appl Compos Mater 26(1):139-157

39. Rukavina T, Ibrahimbegovic A, Kozar I (2019) Fiber-reinforced brittle material fracture models capable of capturing a complete set of failure modes including fiber pull-out. Comput Methods Appl Mech Eng 355:157-192

Publisher's Note Springer Nature remains neutral with regard to jurisdictional claims in published maps and institutional affiliations. 